EPJ Web of Conferences 35, 02005 (2012)

DOI: $10.1051 /$ epjconf/20123502005

(C) Owned by the authors, published by EDP Sciences, 2012

\title{
Charge transport properties of CdMnTe radiation detectors
}

R. Rafiei ${ }^{1, a}$, D. Boardman ${ }^{1}$, M. I. Reinhard ${ }^{1}$, A. Sarbutt ${ }^{1}$, K. Kim ${ }^{2}$, G. C. Watt ${ }^{1}$, S. Uxa ${ }^{3}$, D. A. Prokopovich ${ }^{1}$, E. Belas ${ }^{3}$, A. E. Bolotnikov ${ }^{2}$, and R. B. James ${ }^{2}$

1 Australian Nuclear Science and Technology Organisation, Lucas Heights, NSW, 2234 Australia

2 Brookhaven National Laboratory, Upton, NY 11973 USA

3 Faculty of Mathematics and Physics, Institute of Physics, Charles University, Ke Karlovu 5, Prague 2, CZ-121 16, Czech Republic

\begin{abstract}
Growth, fabrication and characterization of indium-doped cadmium manganese telluride (CdMnTe) radiation detectors have been described. Alpha-particle spectroscopy measurements and time resolved current transient measurements have yielded an average charge collection efficiency approaching $100 \%$. Spatially resolved charge collection efficiency maps have been produced for a range of detector bias voltages. Inhomogeneities in the charge transport of the CdMnTe crystals have been associated with chains of tellurium inclusions within the detector bulk. Further, it has been shown that the role of tellurium inclusions in degrading charge collection is reduced with increasing values of bias voltage. The electron drift velocity was calculated from the rise time distribution of the preamplifier output pulses at each measured bias. From the dependence of drift velocity on applied electric field the electron mobility was found to be $\mu_{n}=(718 \pm 55) \mathrm{cm}^{2} / \mathrm{Vs}$ at room temperature.
\end{abstract}

\section{Introduction}

Semiconductor nuclear radiation detectors are finding increased use in nuclear physics, gamma-ray and X-ray astronomy, medical imaging and national security. Conventional semiconductors consist mainly of germanium and silicon. Their use has however become marginalized in an increasing number of applications due to their physical limitations such as low detection efficiency and the need for cryogenics. Detectors based on high- $Z$ and wide band gap compound semiconductors are logical choices to fulfill this technology need [1]. A high atomic number will give a dominant photoelectric absorption probability, and a wide band gap allows operation at room temperature.

Over the past decade CdTe and CdZnTe compound semiconductors have attracted the most interest in the development of X-ray and gamma-ray detectors and have become commercially available [2]. A very recent and promising candidate is cadmium manganese telluride (CMT) [3]. Its distinct advantages of good compositional homogeneity and a highly tunable band gap compared to $\mathrm{CdZnTe}$ have encouraged CMT detector developments in recent years, bringing large improvements in the performance of CMT as a high resistivity single crystal radiation detector [4-10]. This work describes the growth, fabrication and characterization of indium doped CMT radiation detectors with $5 \%$ manganese concentration $(1.59 \mathrm{eV}$ band gap).

\section{Crystals and Detector Fabrication}

High resistivity $\left(10^{9}-10^{10} \Omega \mathrm{cm}\right)$ single crystals of CMT were grown by the vertical Bridgman technique using a

\footnotetext{
a e-mail: rxr103@gmail.com
}

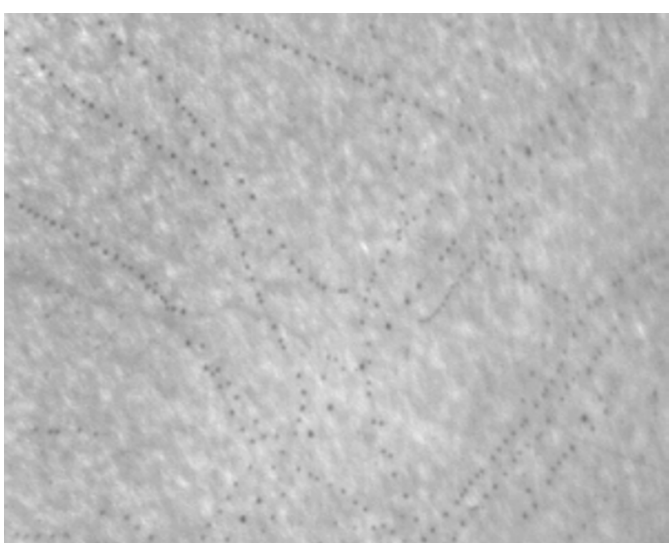

Fig. 1. Infrared image of a $1.2 \times 2.0 \mathrm{~mm}^{2}$ area focal plane beneath the CMT surface. The image shows lines of Te inclusions (dark dots) extending from one side of the crystal to the other.

mixture of CdTe (6N) and MnTe [9]. In order to increase the purity of MnTe source material it was purified twice by the zone-refining method with molten Te. As-grown CMT crystals are p-type and have a low resistivity due to the cadmium vacancies. To ensure the high resistivity of the crystals the ingot was doped with indium at $5 \times 10^{17} \mathrm{~cm}^{-3}$, thus compensating for the high concentration of cadmium vacancies which act as acceptor sites. The ingot was sliced following twins, from which the two samples used for this experiment were cut to the size of $10 \times 10 \times 1.9 \mathrm{~mm}^{3}$. Infrared imaging of each sample revealed networks of tellurium (Te) inclusions as shown in Fig. 1. These inclusions are distributed throughout the bulk at an average concentration of $4.6 \times 10^{5} \mathrm{~cm}^{-3}$, and range in diameter between $1 \mu \mathrm{m}$ and $25 \mu \mathrm{m}$.

The samples were mechanically polished using a $1 \mu \mathrm{m}$ deionized water-based slurry of $\mathrm{Al}_{2} \mathrm{O}_{3}$ which was fol-

This is an Open Access article distributed under the terms of the Creative Commons Attribution License 2.0, which permits unrestricted use, distribution, and reproduction in any medium, provided the original work is properly cited. 


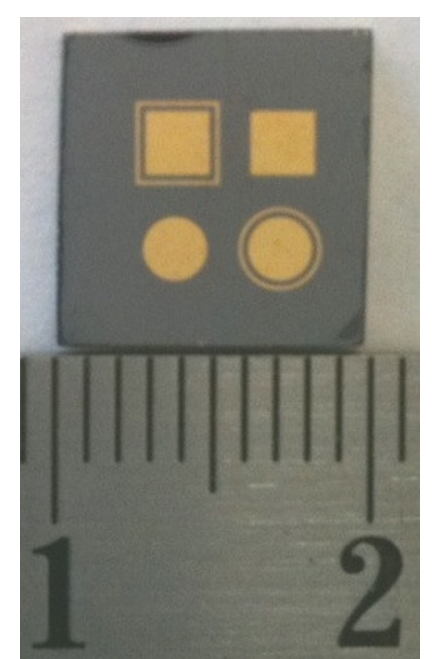

Fig. 2. (Color online) A CMT sample with four front electrode configurations.

lowed by decreasing alumina particle sizes of $0.3 \mu \mathrm{m}$ and $0.05 \mu \mathrm{m}$, respectively. The samples were subsequently etched in a $0.1 \%$ bromine-methanol solution for $5 \mathrm{~min}$ utes. The four electrodes on the front surface of each detector were patterned by photolithography. Metal contacts were deposited by thermal evaporation of $5 \mathrm{~nm} \mathrm{Cr}$ followed by $150 \mathrm{~nm}$ of Au. A single uniform contact covers the rear side. Fig. 2 shows a CMT sample with $2 \mathrm{~mm}$ diameter circular contacts and squares of $2 \mathrm{~mm}$ sides.

\section{Average charge collection efficiency measurements and Hecht analysis}

To obtain the average charge collection efficiency (CCE) across the CMT detectors, both alpha-particle spectroscopy [9] and transient current technique (TCT) [10] measurements were carried out. For these measurements the negative bias was applied to the circular detector (without guard ring) shown in Fig. 2, and each sample was irradiated with $5.48 \mathrm{MeV} \alpha$-particles from an ${ }^{241} \mathrm{Am} 3.9 \mathrm{kBq}$ activity source. As the range $R_{\alpha}=22 \mu \mathrm{m}$ of the $\alpha$-particles in the CMT sample is short compared to the $1900 \mu \mathrm{m}$ detector thickness, the measured signals are almost entirely due to electron drift across the bulk of the detector. The measurements were carried out inside a vacuum chamber at a pressure of less than $5 \times 10^{-5}$ Torr.

For spectroscopy measurements sample B was capacitively coupled to the input of a charge-sensitive Cremat CR-110 preamplifier, which is mounted on a Cremat CR150 test board. The preamplifier output signal was shaped by a CANBERRA 2025 spectroscopy amplifier with a Gaussian shaping time of $2 \mu \mathrm{s}$, followed by acquisition of the pulse height spectra using an Amptek 8000A MCA.

For TCT measurements [10] the signal from sample A was amplified by a Philips Scientific model 6954-B-10 amplifier. This wideband voltage amplifier has a rise time of $180 \mathrm{ps}$ and a bandwidth of $2.3 \mathrm{GHz}$. The amplifier has a specified gain of 10 (measured value of 9.82), an ACcoupled input termination resistor of $50 \Omega$, and is specified to drive a $50 \Omega$ load. The output of the amplifier was digitized at $10 \mathrm{GS} / \mathrm{s}$ using a LeCroy WaveRunner 6100A dig-

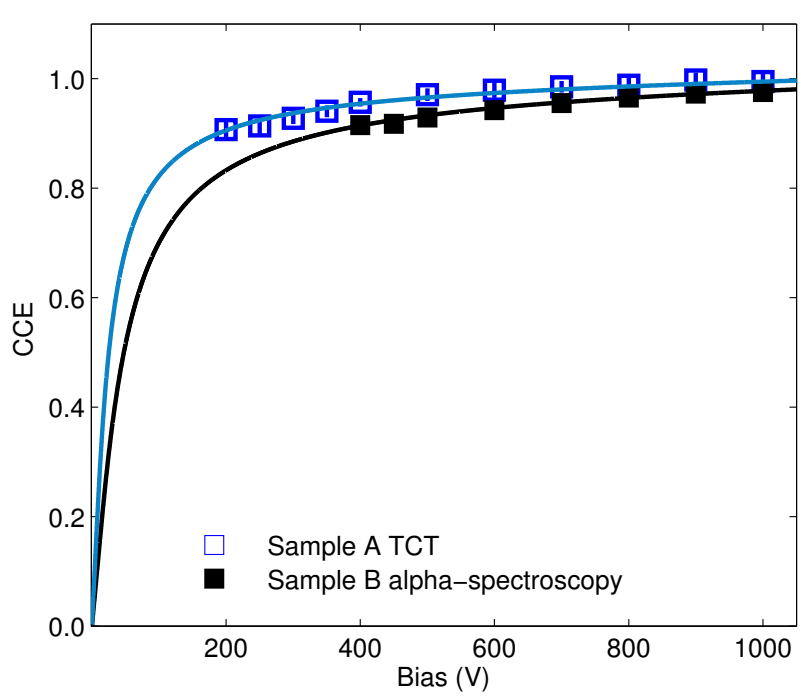

Fig. 3. (Color online) The variation of the average charge collection efficiency across the circular detector on sample A (open squares) and sample B (filled squares) with applied (negative) bias voltage. Error bars are contained within the data points.

ital sampling oscilloscope with an analogue bandwidth of $1 \mathrm{GHz}$.

To obtain the average CCE the alpha induced charge signals from sample B were calibrated using a test pulse and a silicon PIN photodiode with a nominal CCE of $100 \%$. The CCE spectra were corrected for the difference in the electron-hole pair creation energies of $3.6 \mathrm{eV}$ for $\mathrm{Si}$ and $4.29 \mathrm{eV}$ [11] for CMT. The filled squares in Fig. 3 show the dependence of CCE on the applied bias voltage for sample B. For transient current measurements the alpha induced current pulses from sample A were integrated to obtain the induced charge $Q$ which was normalized to the calculated generated total charge $Q_{0}$. The open squares in Fig. 3 show the dependence of CCE on the applied bias voltage for sample A. The average charge collection efficiency across both detectors increases with higher bias values, approaching $100 \%$ for biases above $|V|=1000 \mathrm{~V}$.

The measured data points have been fitted using the Hecht equation which for an induced charge signal due to the drift of electrons alone is reduced to [12]

$$
\mathrm{CCE}=\frac{Q}{Q_{0}}=\frac{\lambda_{n}}{L}+\frac{\lambda_{n}^{2}}{L R_{\alpha}} e^{\frac{-L}{\lambda_{n}}}\left(1-e^{\frac{R_{\alpha}}{\lambda_{n}}}\right) .
$$

The drift length of the electrons is given by $\lambda_{n}=\mu_{n} \tau_{n} \frac{V}{L}$, for an electrode separation of $L$, applied bias $V$, and electron mobility-lifetime product of $\mu_{n} \tau_{n}$. By fitting the model calculation to the measured data an average electron mobilitylifetime product of $\mu_{n} \tau_{n}=(8.5 \pm 0.4) \times 10^{-4} \mathrm{~cm}^{2} \mathrm{~V}^{-1}$ for sample $\mathrm{A}$ and $\mu_{n} \tau_{n}>4.8 \times 10^{-4} \mathrm{~cm}^{2} \mathrm{~V}^{-1}$ for sample $\mathrm{B}$ is estimated. The calculation of a lower limit of $\mu_{n} \tau_{n}$ was only possible for the alpha spectroscopy measurements as excessive broadening of the peaks at biases below $|V|=400 \mathrm{~V}$ increased the Gaussian fitting error used to obtain the centroid of the pulse height spectra and were excluded from the Hecht analysis. When the drift length $\lambda_{n}=4.5 \mathrm{~cm}$ the charge collection efficiency approaches $100 \%$. 
Heavy Ion Accelerator Symposium 2012

\section{Spatially resolved charge collection efficiency measurements}

Ion beam induced charge (IBIC) imaging [13] measurements were carried out to evaluate the CCE as a function of the spatial position across the detectors. For IBIC measurements the microprobe beam line at the Australian Nuclear Science and Technology Organisation was used to focus $5.5 \mathrm{MeV}^{4} \mathrm{He}^{2+}$ beams produced by the ANTARES accelerator down to $\sim 1 \mu \mathrm{m}$ diameter. In this operation the beam was raster scanned over each detector at a count rate between 500 and 1000 ions per second. For each scan, $512 \times 512$ pixel IBIC maps were produced by calculating the median value of the pulse height spectra at each pixel.

Fig. 4 shows a CCE image acquired from sample B at an applied bias of $-400 \mathrm{~V}$. Reduced charge collection regions are spread throughout the detector bulk and are associated with the network of Te inclusions present within the crystal bulk.

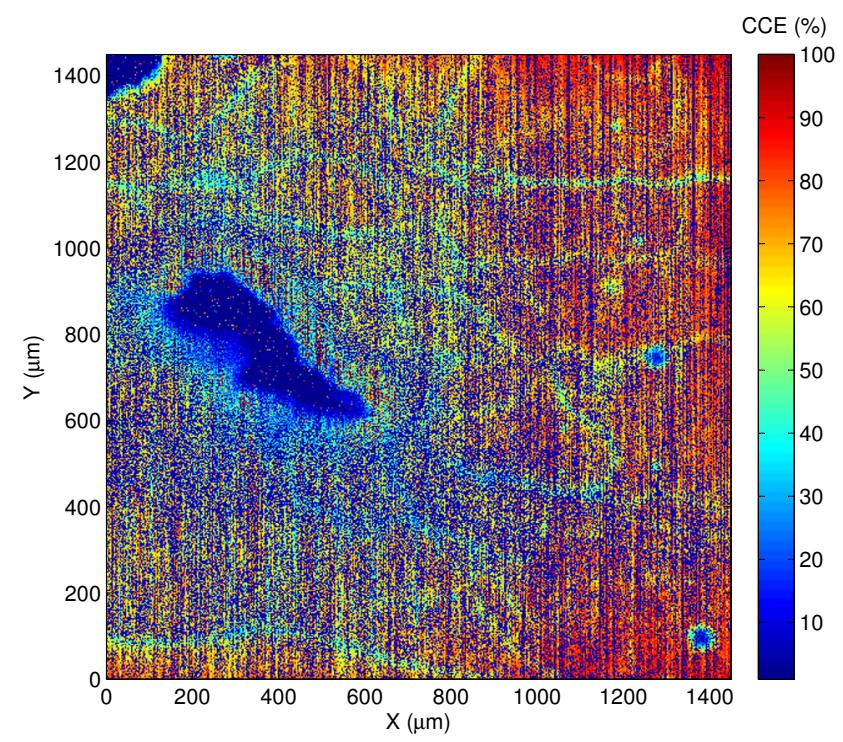

Fig. 4. (Color online) Spatially resolved CCE image of sample B at $-400 \mathrm{~V}$.

The CCE histograms corresponding to measurements made at $-400 \mathrm{~V}$ and $-600 \mathrm{~V}$ are shown in the first and second rows of Fig. 5, respectively. Two peaks are present in each histogram which have been separated by the dashed line and marked as (i) and (ii). IBIC images corresponding to events in (i) and (ii) are presented in the second and third columns, respectively. Variations in image quality are due to the different image collection times. The IBIC map of peak (i) presented in the second column is the reduced $\mathrm{CCE}$ region and is associated with Te inclusions network (sub-grain boundaries) known to act as charge trapping centers in CMT [4] or contributing to the screening of the electric field. The IBIC maps in the third column generated from events in peak (ii) correspond to the higher CCE regions. For the measurement at $-400 \mathrm{~V}$, the $\mathrm{CCE}$ is reduced from a mean value of $76 \%$ to $57 \%$ in the locality of the Te inclusions. Comparing the histograms, with increasing bias values the CCE increases for both (i) and (ii) and the two peaks merge. This means that the influence of the Te inclusions in degrading the performance of the detector is reduced with increasing values of bias voltage, and the detector response uniformity improves.

\section{Carrier transit time and drift mobility measurements}

For the measurement of the transit time of the electrons across sample A the CR-110 preamplifier output pulses were recorded by a LeCroy WaveRunner 6100A digital sampling oscilloscope. The transit time at each measured bias was calculated from the mean of the $10-90 \%$ rise time distribution for a minimum of 2000 recorded pulses. The transit times are shown in the left panel of Fig. 6 and are inversely proportional to the applied bias.

In the right panel of Fig. 6 the drift velocity $v_{n}$ is presented as a function of the applied electric field $E$. The drift velocity reaches full saturation beyond $5500 \mathrm{~V} / \mathrm{cm}$. The room temperature mobility of the CMT sample $\mu_{n}=$ $(718 \pm 55) \mathrm{cm}^{2} / \mathrm{Vs}$ was calculated from a linear regression to the region of proportionality where $v_{n}=\mu_{n} E$ holds. By combining the carrier mobility-lifetime product obtained using the Hecht formalism with the mobility value, the mean electron carrier lifetime was estimated to be $\tau_{n}=(1.2 \pm 0.1) \mu \mathrm{s}$.

\section{Summary}

Studies of charge transport properties of $\mathrm{Cd}_{0.95} \mathrm{Mn}_{0.05} \mathrm{Te}: \mathrm{In}$ semiconductor radiation detectors have been carried out. From $\alpha$-particle spectroscopy and transient current measurements, the detectors approached an average charge collection efficiency of $100 \%$. High resolution maps of charge collection efficiency were measured by scanning $5.5 \mathrm{MeV}$ ${ }^{4} \mathrm{He}^{2+}$ focused beams across the surface of the detectors. The influence of tellurium inclusions on the charge transport performance of CMT detectors has been discussed. It has been shown that the charge collection degradation by Te inclusions declines at higher bias voltages, and the detector's performance becomes more uniform. The dependence of the drift velocity on electric field has been measured and shown to saturate for electric fields greater than $5500 \mathrm{~V} / \mathrm{cm}$. From the region of proportionality the electron mobility was found to be $\mu_{n}=(718 \pm 55) \mathrm{cm}^{2} / \mathrm{Vs}$. Refinements to the CMT crystal growth process, in particular reducing the impurity content of the MnTe source material by repeating the zone-refining process multiple times, are expected to produced CMT radiation detectors with improved charge transport properties.

This work was supported in part by the Cooperative Research Centre for Biomedical Imaging Development. The authors from Brookhaven National Laboratory gratefully acknowledge support from the DOE Office of Nonproliferation Research and Verification, NA22. The authors acknowledge the exceptional technical contributions of N. Zvison and M. Martyniuk from the University of Western Australia in fabricating the CMT detectors. The authors are grateful to the staff of the ANTARES accelerator for providing high quality ${ }^{4} \mathrm{He}$ beams. 

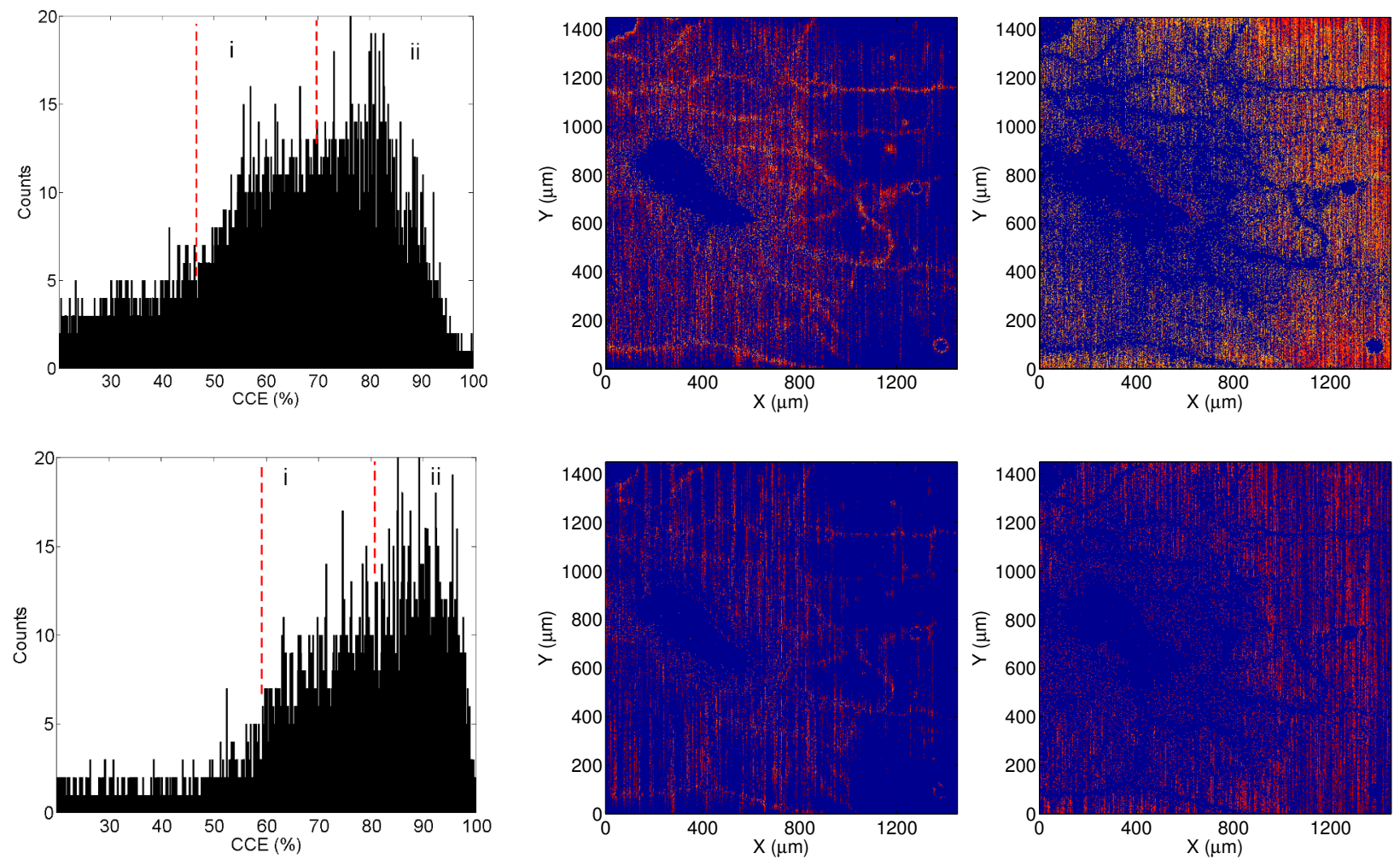

Fig. 5. (Color online) The first column is the histogram of the CCE maps at $-400 \mathrm{~V}$ (top) and $-600 \mathrm{~V}$ (bottom). CCE maps corresponding to peaks marked (i) and (ii) in the first column are presented in columns 2 and 3, respectively. See text for details.
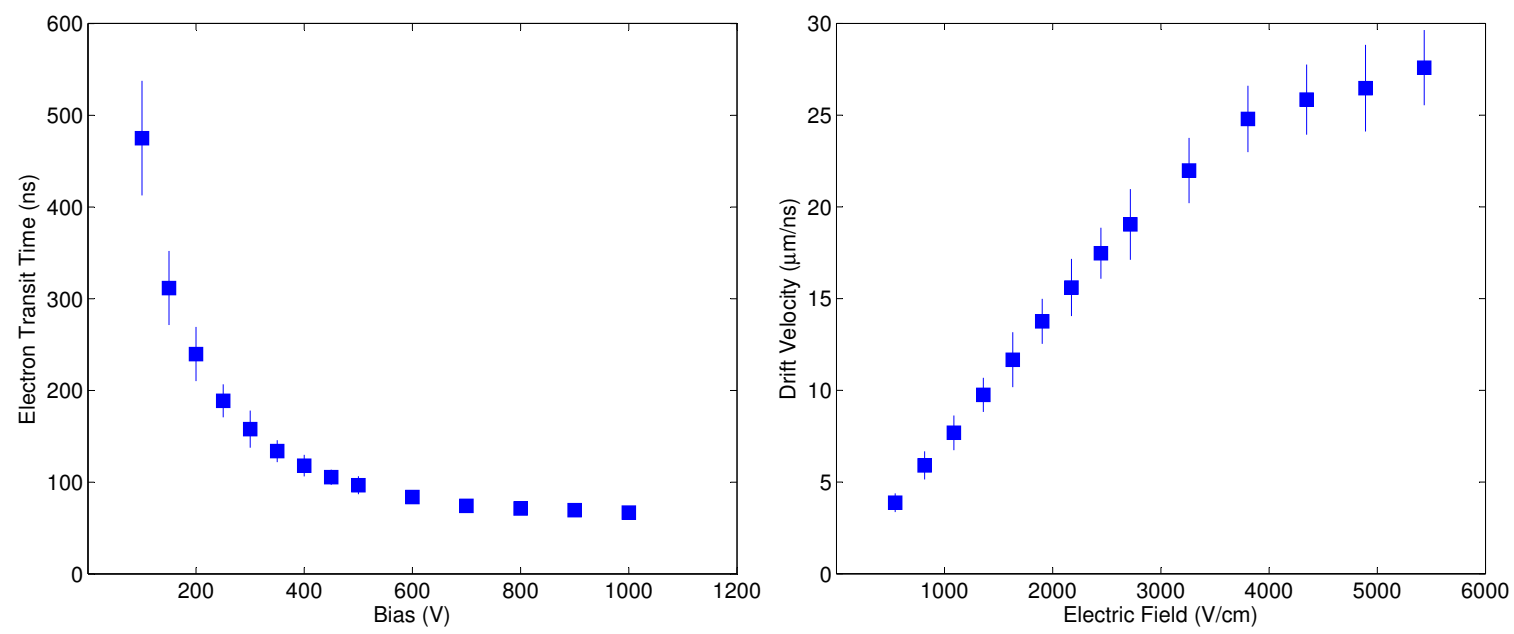

Fig. 6. Bias dependence of the electron transit times (left panel). Electron drift velocity as a function of the applied electric field (right panel).

\section{References}

1. A. Owens and A. Peacock, Nucl. Inst. and Meth. A 531, (2004) 18-37.

2. S. Del Sordo, L. Abbene, E. Caroli, A. M. Mancini, A. Zappettini, and P. Ubertini, Sensors 9, (2009) 34913526.

3. A. Burger et al., J. Cryst. Growth 198/199, (1999) 872876.

4. O. S. Babalola et al., J. Cryst. Growth 311, (2009) 3702.

5. A. Hossain et al., J. Electron. Mater. 38, (2009) 1593.

6. K. Kim, S. Cho, J. Suh, J. Hong, and S. Kim, IEEE Trans. Nucl. Sci. 56, (2009) 858.
7. M. Witkowska-Baran et al., IEEE Trans. Nucl. Sci. 58, (2011) 347.

8. K. H. Kim et al., J. Appl. Phys. 109, (2011) 113715.

9. R. Rafiei et al., IEEE Trans. Nucl. Sci. 59, (2012) 634641.

10. R. Rafiei et al., IEEE Trans. Nucl. Sci., submitted.

11. J. Parkin, Ph.D. Thesis, Faculty of Eng. and Phys. Sci., Univ. Surrey, Surrey, U.K., 2009.

12. Y. Nemirovsky, A. Ruzin, G. Asa, and J. Gorelik, J. Electron. Mater. 25, (1996) 1221.

13. M. B. H. Breese, E. Vittone, G. Vizkelethy, and P. J. Sellin, Nucl. Inst. and Meth. B 264, (2007) 345-360. 